\title{
A Sternum-Disk Distance Method to Identify the Skin Level for Approaching a Surgical Segment without Fluoroscopy Guidance during Anterior Cervical Discectomy And Fusion
}

\author{
Gun Woo Lee ${ }^{1}$, Myun-Whan Ahn ${ }^{1}$, Ji-Hoon Shin ${ }^{1}$, Jae Woo Park ${ }^{1}$, Jae-Hyung $\mathrm{Uh}^{2}$, \\ Jong-Ho Park ${ }^{2}$, Ji-Hoon Lee ${ }^{2}$, Dong-Wook Kim², Jin S. Yeom ${ }^{3}$, Bo-Gun Suh² \\ ${ }^{1}$ Department of Orthopaedic Surgery, Yeungnam University Hospital, Yeungnam University College of Medicine, Daegu, Korea \\ ${ }^{2}$ Spine Center and Department of Orthopaedic Surgery, Pohang Semyeng Christianty Hospital, Pohang, Korea \\ ${ }^{3}$ Spine Center and Department of Orthopaedic Surgery, Seoul National University Bundang Hospital, \\ Seoul National University College of Medicine, Sungnam, Korea
}

Study Design: A retrospective review of prospectively collected data.

Purpose: To introduce the sternum-disk distance (SDD) method for approaching the exact surgical level without C-arm guidance during anterior cervical discectomy and fusion (ACDF) surgery and to evaluate its accuracy and reliability.

Overview of Literature: Although spine surgeons have tried to optimize methods for identifying the skin level for accessing the operative disk level without $\mathrm{C}$-arm guidance during $\mathrm{ACDF}$, success has rarely been reported.

Methods: In total, 103 patients who underwent single-level ACDF surgery with the SDD method were enrolled. The primary outcome measure was the accuracy of the SDD method. The secondary outcome measures were the mean SDD value at each cervical level from the cranial margin of the sternum in the neutral and extension positions of the cervical spine and the inter- and intra-observer reliability of the SDD outcome determined using repeated measurements by three orthopedic spine surgeons.

Results: The SDD accuracy (primary outcome measure) was indicated in 99\% of the patients (102/103). The mean SDD values in the neutral-position magnetic resonance imaging (MRI) were $108.8 \mathrm{~mm}$ at C3-C4, $85.3 \mathrm{~mm}$ at C4-C5, 64.4 $\mathrm{mm}$ at C5-C6, $44.3 \mathrm{~mm}$ at C6$\mathrm{C} 7$, and $24.1 \mathrm{~mm}$ at C7-T1; and those in the extension-position MRI were $112.9 \mathrm{~mm}$ at C3-C4, $88.7 \mathrm{~mm}$ at C4-C5, $67.3 \mathrm{~mm}$ at C5-C6, $46.5 \mathrm{~mm}$ at C6-C7, and $24.3 \mathrm{~mm}$ at C7-T1. The Cohen kappa coefficient value for intra-observer reliability was 0.88 (excellent reliability), and the Fleiss kappa coefficient value for inter-observer reliability as reported by three surgeons was 0.89 (excellent reliability). Conclusions: Based on the results of the present study, we recommend performing ACDF surgery using the SDD method to determine the skin level for approaching the surgical cervical segment without fluoroscopic guidance.

Keywords: Spinal fusion; Cervical vertebrae; Fluoroscopy; Magnetic resonance imaging; Sternum-disk distance method

\footnotetext{
Received May 15, 2016; Revised Jun 9, 2016; Accepted Jun 24, 2016

Corresponding author: Bo-Gun Suh

Spine Center and Department of Orthopaedic Surgery, Pohang Semyeng Christianity Hospital,

351 Posco-daero, Nam-gu, Pohang 37816, Korea

Tel: +82-31-787-6257, Fax: +82-54-289-1765, E-mail: ortho99@naver.com
} 


\section{Introduction}

Anterior cervical discectomy and fusion (ACDF) has been a notably popular surgical option for cervical pathologies that require decompression and fusion [1]. An anterior cervical approach such as ACDF, has several strengths over a posterior approach; however, it can also be detrimental including the possibility of damage to vital organs such as the trachea, esophagus, and critical neurovascular structures and because of the requirement of fluoroscopic guidance for identifying the surgical level. Specifically, for surgical level identification, a posterior cervical approach could be performed accurately without fluoroscopic guidance by conjugating part of the surface anatomy such as the spinous process prominence and peculiarity of the spinous process shape, and evaluating the preoperative images $[1,2]$. However, in the anterior cervical approach, exact identification of the surgical level using surface anatomy such as the hyoid bone and cricoid process, is not always possible because its level can vary based on the patient's surgical position and anatomy [2-5].

Anterior cervical surgery requires identification of the skin level for approaching the exact surgical level, for which most surgeons have to either utilize C-arm fluoroscopy prior to and/or during surgical procedures, or use surface anatomy to initiate the surgical procedure at the predicted point and confirm it during surgery using the $\mathrm{C}$-arm. However, the approach method using fluoroscopic guidance can be problematic. Incorrect determination of the skin level can cause unnecessary soft tissue dissection and lead to increased retraction time of the critical structures, postoperative bleeding or hematoma, neck pain, and discomfort $[4,6-10]$. In addition, using the $\mathrm{C}$-arm can contaminate the surgical field, can be a radiation hazard to the surgeon, and may increase the surgical duration [11]. Therefore, spine surgeons have tried to optimize methods for identifying the skin level to access the operative disk level without the $\mathrm{C}$-arm fluoroscopic guidance during anterior cervical spine surgery.

We devised a novel method that uses preoperative magnetic resonance imaging (MRI), which we designated as the sternum-disk distance (SDD) method. In the present study, we aimed to introduce the SDD method and evaluate its accuracy and reliability for approaching the exact surgical level without $\mathrm{C}$-arm guidance during ACDF. To the best of our knowledge, the present study is a rare investigation of an ideal method for identifying the surgical level without using fluoroscopic guidance during anterior cervical surgery, and it is the first to introduce the novel SDD method.

\section{Materials and Methods}

\section{Patients}

The Institutional Review Board approved the present study. All patients that were scheduled to undergo singlelevel ACDF surgery were enrolled. After January 2014, we performed all ACDF surgeries using the SDD method for identifying the surgical level, based on the results of a pilot trial that positively demonstrated the accuracy of the SDD method. To determine the accuracy and reliability of the SDD method, we retrospectively reviewed the prospectively collected data after January 2014.

The inclusion criteria were: (1) patients with a herniated cervical intervertebral disc and cervical spondylotic radiculopathy and/or myelopathy diagnosed using cervical spine radiographs and magnetic resonance (MR) images that corresponded to clinical manifestations and physical examinations; (2) patients who underwent single-level ACDF; (3) patients between 20 and 80 years of age; and (4) patients who volunteered for the study and provided written consent. The exclusion criteria were: (1) fractures, infection, or tumors in the cervical spine; (2) severe deformity of the cervical spine that required corrective surgery; and (3) a surgical history of operations that involved the sternum.

\section{SDD measurement}

The authors devised a novel SDD method that applied preoperative MRI for identifying the exact surgical level without other aids. First, the cranial portion of the sternum was exposed in the preoperative sagittal MRI, which had to be requested and confirmed with the radiologist prior to the surgery (Fig. 1). All SDD measurements were performed according to the following steps. First, the sagittal and axial MR images were simultaneously viewed on a computer monitor and the two images were linked. Second, the sagittal MR image matching the reference that represented the midline on the axial MR image was obtained. Third, on the sagittal MR image, a line was drawn that perpendicularly extended from the mid-height level of the surgical segment to the skin of the anterior neck 
(Fig. 2A). The bisecting line that is mid-height to the affected disk space on the preoperative sagittal MR image was used as a reference line. Fourth, the distance from the cranial margin of the sternum to the point of the anterior neck skin determined in the third step was calculated and designated as the SDD (Fig. 2B). These measurements were performed by three orthopedic spine surgeons at two different times using a picture archiving and communication system program (Infinitt, Bracknell, Berkshire, UK).

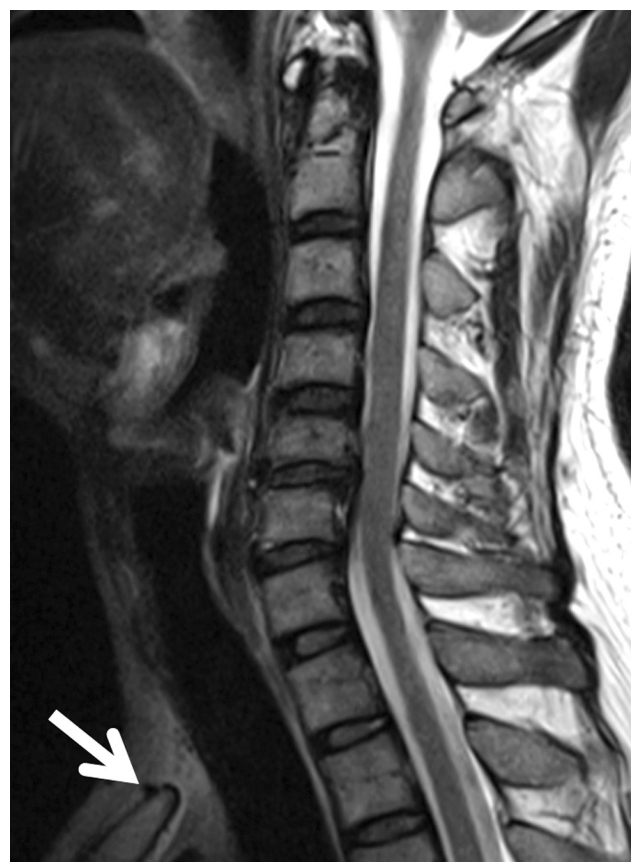

Fig. 1. In the preoperative sagittal magnetic resonance imaging, the cranial portion of the sternum (white arrow) should be exposed.
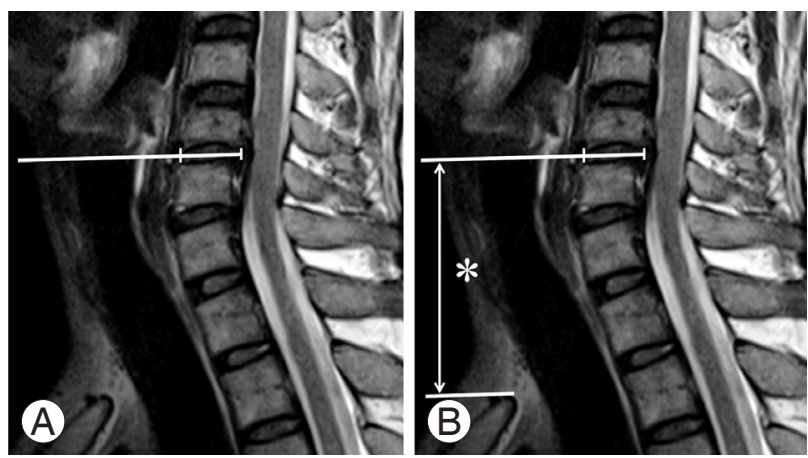

Fig. 2. A C5-C6 cervical segment. (A) On the sagittal MRI image, a line that perpendicularly extended from the mid-height level of the operated segment to the skin of the anterior neck was drawn. (B) The distance from the cranial margin of the sternum to the point of the anterior neck skin determined in the third step was calculated (asterisk), and it was designated as the sternum-disk distance. MRI, magnetic resonance imaging.

\section{Surgical application}

All surgical procedures were performed by the same spine surgeon (the corresponding author) using the same technique. The anterior neck surgical field was sterilized and draped to expose the cranial portion of the sternum. A standard Smith-Robinson procedure was performed at the cervical spine through a transverse incision at the level measured using the SDD method with the help of a ruler. The predicted disk level was exposed, a metal marker was placed on the adjacent vertebral body of the predicted disk level, and lateral fluoroscopy was performed for confirmation. After confirmation, the marker was removed, the endplates were prepared with a quadrangular curette and a pituitary rongeur, and the neural structures were sufficiently decompressed with or without uncoforaminotomy as required. A harvested tricortical iliac bone strut was gently inserted while the interbody space was distracted. Rigid anterior plate-screw fixation (Atlantis, Medtronic, Memphis, TN, USA) was performed in all patients.

\section{Outcome measures}

The primary outcome measure was the accuracy of the SDD method. The measured SDD was applied during actual surgery, and whether the target level could be approached by the SDD without other aids was confirmed by determining the level of the guide pin in a lateral fluoroscopic image as described above. If the predicted disk segment was fluoroscopically confirmed to be at the correct surgical level, we classified it as a "success"; if not, it was classified as a "failure."

The secondary outcome measures were (1) the mean SDD value at each cervical level from the cranial margin of the sternum in the neutral and extension positions of the cervical spine and (2) the inter- and intra-observer reliability values of the SDD outcome. We measured the mean value of the SDD at each level of the cervical spine in the neutral sagittal MRI. In addition, because the SDD can differ depending on the neck position (neutral vs. extension), the SDD value of each neck position and its corresponding differentiation value were also evaluated. SDD was measured independently by three orthopedic spine surgeons in order to determine the inter-observer reliability, and the measurements were repeated using the same radiographs one month after the initial measurement in order to analyze the intra-observer reliability and 
to mitigate recall bias. The reviewers were blinded to the information on the previous results during the second measurement. All measurement data were independently tabulated by another blinded orthopedic surgeon.

\section{Statistical analysis}

The intra-observer reliability for each value reported by the three observers was analyzed using the corresponding Cohen kappa coefficients and the inter-observer reliability was assessed using the corresponding Fleiss generalized kappa coefficients. The strengths of the inter- and intraobserver reliabilities were determined using the study of Landis and Koch $[12,13]$, where kappa values of $0.21-0.40$ indicated fair reliability; $0.41-0.60$ indicated moderate reliability; $0.61-0.80$ indicated substantial reliability; and $0.81-1.0$ indicated excellent reliability. All statistical analyses of inter- and intra-observer reliability were performed using the MedCalc software (MedCalc 12.3.0.0, Mariakerke, Belgium). The level of significance was set at a 2 -tailed $p<0.05$.

\section{Results}

\section{Population}

In total, 103 patients who satisfied the study inclusion criteria were enrolled in the present study and their demographic data are described in Table 1.

\section{Outcomes}

The SDD accuracy (primary outcome measure) indicated that in $99 \%$ of the patients (102/103), the exact level was approached using the SDD method. At the cervical level, the accuracy was $100 \%$ at C3-C4 (2/2), 100\% at C4-C5 (18/18), $100 \%$ at C5-C6 (46/46), $97.1 \%$ at C6-C7 (33/34), and $100 \%$ at $\mathrm{C} 7-\mathrm{T} 1(3 / 3)$. The assessment of the approach (left $v s$. right) showed that the accuracy of the right-sided approach was $100 \%(31 / 31)$ and the accuracy of the leftsided approach was $98.6 \%(71 / 72)$.

The mean SDD value at each cervical segment from the upper margin of the sternum was evaluated in a neutralposition MRI as follows: $108.8 \mathrm{~mm}$ at $\mathrm{C} 3-\mathrm{C} 4,85.3 \mathrm{~mm}$ at $\mathrm{C} 4-\mathrm{C} 5,64.4 \mathrm{~mm}$ at $\mathrm{C} 5-\mathrm{C} 6,44.3 \mathrm{~mm}$ at $\mathrm{C} 6-\mathrm{C} 7$, and 24.1 $\mathrm{mm}$ at $\mathrm{C} 7-\mathrm{T} 1$. The mean SDD value at each cervical segment in an extension-position MRI was evaluated as fol-
Table 1. Demographic data

\begin{tabular}{lc} 
Variables & Patients \\
\hline Case $(\mathrm{n})$ & 103 \\
\hline Age $(\mathrm{yr})$ & $54.8 \pm 11.6$ \\
\hline Sex (male/female) & $58 / 45$ \\
\hline Height $(\mathrm{cm})$ & $164.0 \pm 8.9$ \\
\hline Weight $(\mathrm{kg})$ & $63.8 \pm 10.1$ \\
\hline Body mass index $\left(\mathrm{kg} / \mathrm{m}^{2}\right)$ & $23.8 \pm 3.0$ \\
\hline Surgical level & \\
\hline C3-4 $(\%)$ & $2(2)$ \\
\hline C4-5 $(\%)$ & $18(17)$ \\
\hline C5-6 $(\%)$ & $46(45)$ \\
\hline C6-7 $(\%)$ & $34(33)$ \\
\hline C7-T1 $(\%)$ & $3(3)$ \\
\hline Approach side & \\
\hline Left & $72(70)$ \\
\hline Right & $31(30)$ \\
\hline
\end{tabular}

Values are presented as mean \pm standard deviation or number (\%).

lows: $112.9 \mathrm{~mm}$ at $\mathrm{C} 3-\mathrm{C} 4,88.7 \mathrm{~mm}$ at $\mathrm{C} 4-\mathrm{C} 5,67.3 \mathrm{~mm}$ at $\mathrm{C} 5-\mathrm{C} 6,46.5 \mathrm{~mm}$ at $\mathrm{C} 6-\mathrm{C} 7$, and $24.3 \mathrm{~mm}$ at $\mathrm{C} 7-\mathrm{T} 1$. The differences between the SDD values in the neutral- and extension-position MRIs at each cervical segment were 4.1 $\mathrm{mm}$ at $\mathrm{C} 3-\mathrm{C} 4,3.4 \mathrm{~mm}$ at $\mathrm{C} 4-\mathrm{C} 5,2.9 \mathrm{~mm}$ at C5-C6, 2.2 $\mathrm{mm}$ at $\mathrm{C} 6-\mathrm{C} 7$, and $0.2 \mathrm{~mm}$ at C7-T1 (Table 2).

The Cohen kappa coefficients of the intra-observer reliability are shown in Table 3; the mean kappa coefficient value was 0.88 , indicating "excellent" reliability. The Fleiss kappa coefficients of the inter-observer reliability among the three observers are shown in Table 3; the mean Fleiss coefficient value was 0.89 , indicating "excellent" reliability.

\section{Discussion}

The difficulty of determining the skin level for approaching the exact surgical segment during anterior cervical surgery such as ACDF without using fluoroscopic guidance is a critical issue, and most spine surgeons must utilize C-arm fluoroscopy prior to and/or during surgical procedures to confirm that the skin level is correct [1-3]. However, fluoroscopic guidance can be accompanied by radiation hazards and can create contamination issues for surgeons and patients. Therefore, we developed the SDD method as a novel approach for determining the skin level without fluoroscopic guidance for assessing the operative 
Table 2. SDD value (mm) of each cervical level according to neck position

\begin{tabular}{lrrrr} 
Level & Neutal & Extension & Differentiation $^{\text {a) }}$ & Mean disc height \\
C3-4 & $108.8 \pm 5.4$ & $112.9 \pm 5.9$ & 4.1 & 5.3 \\
C4-5 & $85.3 \pm 3.1$ & $88.7 \pm 3.5$ & 3.4 & 5.6 \\
C5-6 & $64.4 \pm 3.9$ & $67.3 \pm 3.5$ & 2.9 & 5.4 \\
C6-7 & $44.3 \pm 2.6$ & $46.5 \pm 2.7$ & 2.2 & 5.6 \\
C7-T1 & $24.1 \pm 3.4$ & $24.3 \pm 3.3$ & 0.2 & 5.5 \\
\hline
\end{tabular}

Values are presented as mean \pm standard deviation or number (\%).

SDD, sternum-disk distance.

alExtension value-Neutral value.

Table 3. Cohen's Kappa coefficients for intra-observer reliability and fleiss kappa coefficients for inter-observer reliability of SDD method ( $\mathrm{n}=103$ )

\begin{tabular}{lcccc} 
Time & Observer A & Observer B & Observer C & Mean \\
First $^{\mathrm{a})}$ & $0.88(0.83,0.95)$ & $0.91(0.86,0.93)$ & $0.87(0.83,0.95)$ & 0.89 \\
Second $^{\text {a) }}$ & $0.87(0.85,0.91)$ & $0.90(0.86,0.96)$ & $0.89(0.84,0.92)$ & 0.88 \\
Mean & 0.87 & 0.90 & 0.88 & - \\
\hline
\end{tabular}

SDD, sternum-disk distance.

${ }^{\text {al }}$ The numbers in parentheses indicate the $95 \%$ confidence interval.

segment during anterior cervical surgery.

In the present study, we evaluated the accuracy of the SDD method, and calculated the mean SDD value for each cervical segment according to neck position and the inter- and intra-observer reliability of this value. The accuracy of the SDD method was $99 \%$ (102/103). The single failed case involved C6-C7 with a left-sided approach; in this case the surgery was performed with the patient at an incorrect position (the Trendelenburg posture) for intubation that the operator and others were unaware of. With the exception of this single case, we demonstrated that the SDD method is a promising option for accessing the exact cervical disk level without fluoroscopic guidance. In addition, we analyzed the intra- and inter-observer reliability of the SDD method with repeated measurements by three orthopedic surgeons. The Cohen kappa coefficient value was 0.88 (excellent reliability), while the Fleiss kappa coefficient value among the three observers was 0.89 (excellent reliability). The coefficient values indicated that the SDD measurement method demonstrated excellent consistency irrespective of the time of measurement and the person who performed the measurement.

In addition, we measured the mean SDD value for each cervical segment and the cranial margin of the sternum in a neutral-position MRI, as described at the Results section. However, the anterior cervical surgery was some- times performed with a slightly extended neck position, which can introduce measurement errors. Therefore, we also measured the mean SDD value in an extension-position MRI and calculated the difference between the values measured during the neutral- and the extension-position MRIs. The differentiation between the neck positions (neutral vs. extension) can be critical for measuring the SDD method error margin. However, in the present study, the mean disk height at the surgical segments of all patients was $5.53 \pm 0.96 \mathrm{~mm}$ (Table 2). This indicated that the differences between neck positions were within the disk height of the surgical level and that the neck position was not a critical factor that influenced the results. The average SDD values measured in the neutral- and extensionposition MRIs were used for the surgeries; these values could be ideal for mitigating measurement errors.

Reports on methods that do not use C-arm guidance are limited. Auerbach et al. [4] reported a novel method that used the mandibular angle as a landmark for identifying the cervical spinal level; however, this method has several limitations: (1) in our experience, there were large differences between neck positions (neutral vs. extension); (2) the study was conducted with a small sample size of only 30 patients; and (3) inter- and intra-observer reliability tests were not performed. Our study had significant advantages over Auerbach's study. First, the results 
of the SDD method were relatively consistent irrespective of the neck position, and the difference between the neutral and the extension positions of the neck was trivial as it was within the disc height of the operative level. In addition, we conducted the present study with a relatively large sample size of 103 patients, and the accuracy of the SDD method was $99 \%$. Finally, we performed inter- and intra-observer reliability assessments and showed excellent intra- and inter-observer agreement ( 0.88 and 0.89 , respectively). Based on the results of the present study, we recommend performing ACDF surgery using the SDD method to determine the skin level for approaching the surgical cervical segment without fluoroscopic guidance.

The present study had several limitations. First, the study was conducted in patients undergoing single-level ACDF surgery as a pilot study of the SDD method. We applied the SDD method in patients with more than two affected cervical levels and used a mid-height bisecting line located between the upper endplate line of the uppermost operative disc space and the lower endplate line of the lowermost operative disc space for reference; however, in these cases the SDD method also showed excellent outcomes in terms of accuracy and reliability. Second, the study was conducted in a relatively small sample size of 103 patients. Additional studies are required to further assess the accuracy and reliability of the SDD method. Finally, the SDD method could not be applied in a patient whose cranial portion of the sternum could not be exposed at the preoperative MRI; however, this problem was inevitable since the cranial sternum was a reference point for measuring the SDD.

\section{Conclusions}

In the present study, we introduced the SDD method as a novel method for determining the skin level to approach the exact surgical cervical segment during ACDF surgery and evaluated the accuracy and reliability of this method. The accuracy of the SDD method (primary outcome measure) was $99 \%$ (102/103 patients). The intra- and inter-observer reliabilities of the SDD method were excellent ( 0.88 and 0.89 , respectively). The results using this method were relatively consistent irrespective of the neck position, and the differences between the neutral and the extension positions of the neck were within the operative level disc height. Therefore, based on the results of the present study, we recommend using the SDD method for determining the skin level to approach the surgical cervical segment during ACDF surgery as it eliminates the need for additional fluoroscopic guidance.

\section{Conflict of Interest}

No potential conflict of interest relevant to this article was reported.

\section{References}

1. Song KJ, Choi BY. Current concepts of anterior cervical discectomy and fusion: a review of literature. Asian Spine J 2014;8:531-9.

2. Hoff JT, Wilson CB. Microsurgical approach to the anterior cervical spine and spinal cord. Clin Neurosurg 1979;26:513-28.

3. Hoppenfeld S, Hutton R. Physical examination of the spine and extremities. New York: Appleton-CenturyCrofts; 1976.

4. Auerbach JD, Weidner Z, Pill SG, Mehta S, Chin KR. The mandibular angle as a landmark for identification of cervical spinal level. Spine (Phila Pa 1976) 2009;34: 1006-11.

5. Cha YD, Lee SK, Kim TJ, Han TH. The neck crease as a landmark of Chassaignac's tubercle in stellate ganglion block: anatomical and radiological evaluation. Acta Anaesthesiol Scand 2002;46:100-2.

6. Cho SK, Lu Y, Lee DH. Dysphagia following anterior cervical spinal surgery: a systematic review. Bone Joint J 2013;95B:868-73.

7. Halani SH, Baum GR, Riley JP, et al. Esophageal perforation after anterior cervical spine surgery: a systematic review of the literature. J Neurosurg Spine 2016;25:285-91.

8. Erwood MS, Hadley MN, Gordon AS, Carroll WR, Agee BS, Walters BC. Recurrent laryngeal nerve injury following reoperative anterior cervical discectomy and fusion: a meta-analysis. J Neurosurg Spine 2016; 25:198-204.

9. Koreckij TD, Davidson AA, Baker KC, Park DK. Retropharyngeal steroids and dysphagia following multilevel anterior cervical surgery. Spine (Phila Pa 1976) 2016;41:E530-4.

10. Song KJ, Choi BW, Kim JK. Adjacent segment pathology following anterior decompression and fusion using cage and plate for the treatment of degenerative 
cervical spinal diseases. Asian Spine J 2014;8:720-8.

11. Racadio JM, Nachabe R, Homan R, Schierling R, Racadio JM, Babic D. Augmented reality on a C-arm system: a preclinical assessment for percutaneous needle localization. Radiology 2016;281:249-55.

12. Landis JR, Koch GG. An application of hierarchical kappa-type statistics in the assessment of majority agreement among multiple observers. Biometrics 1977;33:363-74.

13. Landis JR, Koch GG. The measurement of observer agreement for categorical data. Biometrics 1977;33: 159-74. 